\title{
Schlussbetrachtungen des Tagungsleiters P. Brechtbühl ${ }^{1}$
}

Ich möchte abschliessend drei Feststellungen machen:

1. Aus den Referaten und Diskussionen kann abgeleitet werden, dass Präventivmassnahmen und ihre Aktivierung unbestritten sind.

2. Zu einer harten Kontroverse über die Standorte hier Kurativmedizin

da Präventivmedizin

ist es nicht gekommen. Die These, wonach die Förderung der Präventivmedizin die Effizienz des Gesundheitswesens - allenfalls gar mit Kosteneinsparungen bei der Kurativmedizin - erhöhen würde, scheint allgemein anerkannt zu werden.

3. Persönlich am meisten gepackt haben mich die Referate und Voten zum Problemkreis

hier Staat,

da der mehr oder weniger mündige Bürger

oder

hier obrigkeitlicher Zwang,

da Freiheit und Verantwortung des Bürgers.

Wieweit soll die Betreuungsfunktion des Staates gehen, und welcher Freiheits- und Verantwortungsspielraum soll dem Bürger belassen werden? Noch pointierter gesagt: Gibt es da eine «Betreuungs-Erwartungs-Mentalität», die den Menschen daran hindert oder ihn darin erschlaffen lässt, aus eigener Initiative Verhaltensregeln der Prävention zu befolgen?

Diese heiklen Fragen der Grenzziehung stehen für mich in engem Zusammenhang mit den hochaktuellen Problemen der Sozialpolitik, wobei ich auf ein seltsames Paradoxon unserer Verfassung hinweisen möchte: Wenn die verdienten Väter unserer Bundesverfassung im Text von 1848 die Forderungen nach "persönlicher Freiheit des Bürgers» (gemeint war unter anderem auch die Freiheit des Eigentums) und nach «Beförderung der gemeinsamen Wohlfahrt», erhoben, so gaben sie sich wohl kaum darüber Rechenschaft, dass diese beiden Begriffe dereinst von

\footnotetext{
1 Referat gehalten an der Tagung des Forum Davos 78: Grenzen der Medizin III: Prävention und ihre Möglichkeiten.
}

der Frage des Masses und der Abgrenzung her in Konkurrenz zueinander geraten könnten.

Deutlicher gesagt: Wieviel persönliche Freiheit verbleibt dem Bürger noch, wenn man mit ihm zuviel gemeinsame Wohlfahrt betreibt?

Man kann andersherum fragen: Sind sich die Kreise, die nach fortgesetztem Ausbau des Wohlfahrtsstaates und nach neuen Versicherungsobligatorien rufen, darüber im klaren, dass mit jedem Schritt, der in dieser Richtung getan wird, im gleichen Ausmass die Selbstverantwortung des einzelnen zurückgedrängt wird?

Für mich ist eine wichtige Frage offengeblieben, nämlich die, ob wir geplante Massnahmen der Prävention mit rationalen Kosten/Nutzen-Rechnungen angehen können. Man geht davon aus, dass mit Prävention Krankheiten ausbleiben. Man wird dafür aber immer in einen gewissen Beweisnotstand geraten, weil der Gegenbeweis oftmals fehlt, insbesondere bei Verhaltensfragen. (Beweiskräftig sind Massnahmen der Impfung, der Bekämpfung der Zahnkaries usw.) Bei vielen andern Störfaktoren und Lebensfeinden dagegen sind die Grenzen verwischt. Wie verhält es sich weiter mit der Kostenrechnung, wenn es zwar gelingt, durch Präventiveinwirkung zahlreiche Menschen zwischen 50 und 60 vor einem Herzinfarkt zu bewahren, dafür aber viele dieser Menschen zwischen 65 und 75 durch schwere Krebsleiden während Jahren dahinvegetieren müssen? Darin liegt viel Irrationales.

Ich meine, dass die Arbeiten dieses Kongresses uns bei der Suche nach diesen Abgrenzungen um einige Erkenntnisse weitergebracht haben. Wir wissen aber: Präventivmassnahmen müssen auf lange Frist angelegt werden, und man darf den Schnauf und die Geduld nicht verlieren.

In der zuversichtlichen Erwartung, dass allen verdienten Kämpfern um Aktivierung der Prävention der Schnauf nicht ausgeht, erlaube ich mir, Herrn Bundesrat Hürlimann zu bitten, unsern Kongress mit seiner Ansprache abzuschliessen. 